\title{
MACHO DETECTION
}

\section{FROM MEASUREMENTS OF GRAVITATIONAL DEFLECTION}

\author{
KOUJI OHNISHI \\ Nagano National College of Technology \\ Nagano 381-8550, Japan \\ MIZUHIKO HOSOKAWA \\ C'ommunications Research Laboratory \\ 4-2-1 Nukui-kita,Koganei,Tokyo 184, Japan \\ AND \\ TOSHIO FUKUSHIMA \\ National Astronomical Observatory \\ 2-21-1, Ohsawa,Mitaka,Tokyo 181, Japan
}

The observed positions of quasars are fluctuated due to the gravitational lensing of the matters in our galaxy. The magnitude of fluctuation due to stars and MACHOs is of the order of a few micro-arc second ( $\mu$ as) $\sim 10 \mu$ as and its time scale is of the order of a few years $\sim$ hundreds years (Hosokawa et al.1997). Such fluctuation will reflects the nature of the constituents, both visible and invisible, of our galaxy.

We calculate the probabilities (optical depths) of such fluctuation (i.e. positional shift and apparent proper motion of the quasars ) as the functions of the parameters of the galaxy model that consist of stars and MACHOs. The observation of hundreds of quasar's position with a few $\mu$ as accuracy for several years will enable us to estimate the density profile of galactic MACHO halo (i.e. core radius and axis ratio of spheroidal equipotential) . The possibility to obtain such accuracy is now began to consider seriously in some VLBI and optical interferometer plans (Sasao et al. 1994).

\section{References}

Hosokawa, M., Ohnishi, K., \& Fukushima, 1997, AJ, 114, 1508

Sasao, T., et.al., 1994, VLBI Technology, ed. T. Sasao, S. Manabe, O. Kameya \& M. Inoue, (Terra Scientific Publ. Co.), 254 